\title{
АНАЛИЗ МАКРОБЛОКОВ В ЗАДАЧЕ ОБНАРУЖЕНИЯ АРТЕФАКТОВ ПОТЕРИ ДАННЫХ В ВИДЕО
}

\section{ANALYSIS OF MACROBLOCS IN THE PROBLEM OF DETECTING ARTIFACTS OF DATA LOSS IN VIDEO}

\author{
A. Ustin \\ S. Fedosin \\ M. Sokolova
}

Summary. The article analyzes the H.264 MPEG-4 Part 10 video codec in the context of finding solutions to simplify the detection of data loss artifacts in video materials. Due to the high resource consumption of both the algorithmic approach and the neural network, an attempt was made to reduce these costs by obtaining and processing additional information obtained directly from the structure of the video codec. The article focuses on the use of a macroblock table to speed up the algorithms for detecting data loss artifacts.

Keywords: video analysis, quality assessment, video, artifacts, data loss, packet loss, h264.

\author{
Устин Антон Михайлович \\ Аспирант, Национальный исследовательский \\ Мордовский государственный университет имени \\ Н.П. Огарёва, Саранск \\ ustinam95@yandex.ru \\ Федосин Сергей Алексеевич \\ К.т.н., профессор, Начиональный исследовательский \\ Мордовский государственный университет имени \\ Н.П. Огарёва, Саранск \\ fedosinsa@mrsu.ru \\ Соколова Мария Сергеевна \\ Аспирант, Национальный исследовательский \\ Мордовский государственный университет имени \\ Н.П. Огарёва, Саранск \\ Manunia131313@mail.ru
}

Аннотация. В статье проведен анализ видеокодека H.264 MPEG-4 Part 10 в контексте поиска решений для упрощения обнаружения артефактов потери данных в видео материалах. В связи с высокой ресурсозатратностью как алгоритмического подхода, так и нейросетевого, была предпринята попытка снизить эти расходы путем получения и обработки дополнительной информации, получаемой непосредственно из структуры видео кодека. В статье особое внимание уделяется использованию таблицы макроблоков для ускорения работы алгоритмов обнаружения артефактов потери данных.

Ключевые слова: анализ видео, оценка качества, видео, артефакты потери данных, артефакты потери пакетов, h264.

\section{Введение}

$\mathbf{H}$ а практике для передачи цифрового видео его предварительно сжимают для того, чтобы подстроиться под пропускную способность сети и для эффективного хранения. Обычно используются методы кодирования видео с потерями, что приводят к деградации качества видео, что называют искажением источника. Помимо того, в процессе передачи видео его качество может быть снижено из-за ошибок передачи, в частности из-за потери пакетов. Этот тип артефактов видео называется искажением канала, при этом проявляются типичные визуальные характеристики, которые принципиально отличаются от артефактов искажения источника. Степень искажения канала зависит от множества различных факторов: от количества потерянных пакетов, размеров повреждений в них, от самого видеоконтента, а также от параметров кодирования и самого используемого метода кодирования материалов. В ко-

нечном итоге все это влияет на визуальное качество, воспринимаемое конечным пользователем.

Обнаружение искажений канала может быть полезно как при адаптивной потоковой передачи (например, при ретрансляции поврежденные пакеты могут быть запрошены повторно), так и при анализе локальных архивов видеоматериалов, для их последующего исправления и восстановления при обнаружении проблем.

И если при сетевой передаче в теории у пользователя или у оборудования может быть доступ к сетевой статистике, на основе которой можно делать предположение о потери пакетов для запроса повторной отправки, то для локальных архивов и для случаев с отсутствием информации о сетевом соединении такой возможности может не быть. В таких случаях может подойти сценарий анализа самого видеопотока с поиском и обнаружением артефактов непосредственно в нём. 


\begin{tabular}{|l|l|l|l|l|l|l|l|l|l|l|l|l|l|l|l|l|}
\hline $\mathbf{S}$ & $\mathbf{S}$ & $\mathbf{S}$ & $\mathbf{S}$ & $\mathbf{S}$ & $\mathbf{S}$ & $\mathbf{S}$ & $\mathrm{P}$ & $\mathrm{PB}$ & $\mathrm{PB}$ & $\mathbf{S}$ & $\mathbf{S}$ & $\mathbf{S}$ & $\mathbf{S}$ & $\mathbf{S}$ & $\mathbf{S}$ & $\mathbf{S}$ \\
\hline $\mathbf{S}$ & $\mathbf{S}$ & $\mathbf{S}$ & $\mathbf{S}$ & $\mathbf{S}$ & $\mathbf{S}$ & $\mathbf{S}$ & $\mathrm{PP}$ & $\mathrm{PP}$ & $\mathrm{PB}$ & $\mathbf{S}$ & $\mathbf{S}$ & $\mathbf{S}$ & $\mathbf{S}$ & $\mathbf{S}$ & $\mathbf{S}$ & $\mathbf{S}$ \\
\hline $\mathbf{S}$ & $\mathbf{S}$ & $\mathbf{S}$ & $\mathbf{S}$ & $\mathbf{S}$ & $\mathbf{S}$ & $\mathrm{PP}$ & $\mathrm{PP}$ & $\mathrm{PP}$ & $\mathrm{PB}$ & $\mathbf{S}$ & $\mathbf{S}$ & $\mathbf{S}$ & $\mathbf{S}$ & $\mathbf{S}$ & $\mathbf{S}$ & $\mathbf{S}$ \\
\hline $\mathbf{S}$ & $\mathbf{S}$ & $\mathbf{S}$ & $\mathbf{S}$ & $\mathbf{S}$ & $\mathbf{S}$ & $\mathrm{PP}$ & $\mathrm{PP}$ & $\mathrm{PP}$ & $\mathrm{PB}$ & $\mathbf{S}$ & $\mathbf{S}$ & $\mathbf{S}$ & $\mathbf{S}$ & $\mathbf{S}$ & $\mathbf{S}$ & $\mathbf{S}$ \\
\hline $\mathbf{S}$ & $\mathbf{S}$ & $\mathbf{S}$ & $\mathbf{S}$ & $\mathbf{S}$ & $\mathbf{S}$ & $\mathrm{PB}$ & $\mathrm{PP}$ & $\mathrm{PP}$ & $\mathrm{PB}$ & $\mathrm{PP}$ & $\mathbf{S}$ & $\mathbf{S}$ & $\mathbf{S}$ & $\mathbf{S}$ & $\mathbf{S}$ & $\mathbf{S}$ \\
\hline $\mathbf{S}$ & $\mathbf{S}$ & $\mathbf{S}$ & $\mathbf{S}$ & $\mathbf{S}$ & $\mathbf{S}$ & $\mathrm{PP}$ & $\mathrm{PB}$ & $\mathrm{PP}$ & $\mathrm{PP}$ & $\mathbf{I}$ & $\mathbf{S}$ & $\mathbf{S}$ & $\mathbf{S}$ & $\mathbf{S}$ & $\mathbf{S}$ & $\mathbf{S}$ \\
\hline $\mathbf{S}$ & $\mathbf{S}$ & $\mathbf{S}$ & $\mathbf{S}$ & $\mathbf{S}$ & $\mathbf{S}$ & $\mathbf{S}$ & $\mathrm{PP}$ & $\mathrm{PB}$ & $\mathrm{PB}$ & $\mathbf{S}$ & $\mathbf{S}$ & $\mathbf{S}$ & $\mathbf{S}$ & $\mathbf{S}$ & $\mathbf{S}$ & $\mathbf{S}$ \\
\hline $\mathbf{S}$ & $\mathbf{S}$ & $\mathbf{S}$ & $\mathbf{S}$ & $\mathbf{S}$ & $\mathbf{S}$ & $\mathrm{PP}$ & $\mathrm{PP}$ & $\mathrm{PP}$ & $\mathrm{PP}$ & $\mathbf{I}$ & $\mathbf{S}$ & $\mathbf{S}$ & $\mathbf{S}$ & $\mathbf{S}$ & $\mathbf{S}$ & $\mathbf{S}$ \\
\hline $\mathbf{S}$ & $\mathbf{S}$ & $\mathbf{S}$ & $\mathbf{S}$ & $\mathbf{S}$ & $\mathbf{S}$ & $\mathrm{PP}$ & $\mathrm{PP}$ & $\mathrm{PP}$ & $\mathrm{PP}$ & $\mathrm{PP}$ & $\mathbf{S}$ & $\mathbf{S}$ & $\mathbf{S}$ & $\mathbf{S}$ & $\mathbf{S}$ & $\mathbf{S}$ \\
\hline $\mathbf{S}$ & $\mathbf{S}$ & $\mathbf{S}$ & $\mathbf{S}$ & $\mathbf{S}$ & $\mathbf{S}$ & $\mathrm{PP}$ & $\mathrm{PP}$ & $\mathrm{PP}$ & $\mathrm{PP}$ & $\mathrm{PP}$ & $\mathbf{S}$ & $\mathbf{S}$ & $\mathbf{S}$ & $\mathbf{S}$ & $\mathbf{S}$ & $\mathbf{S}$ \\
\hline
\end{tabular}

Рис. 1. Пример таблицы макроблоков. S - макроблоки типа «Skip», PP, PB, PI — макроблоки, которые используют информацию из различных кадров, I - макроблоки исходного кадра

В последние годы, в связи с быстрым ростом медиаконтента, растет и потребность компаний в алгоритмах оценке качества и поиска артефактов в видео. Особым спросом пользуется класс не ссылочных (Non-Reference) алгоритмов, которым не требуется исходное видео для анализа текущего. Традиционные алгоритмы этого класса основаны на наборах числовых функций, которые объединяют для получения итогового индекса качества изображения. Еще в этом классе алгоритмов на данный момент все больше набирает популярность машинное обучение, которое не только эффективно находит индексы качества, но и находит, а также исправляет различных артефакты изображений. Все эти алгоритмы объединяет оценка пространственных особенностей изображений, таких как локальные разрывы блоков, нарушение распределений или временных паттернов цветов пикселей на изображениях. Все это требует значительных ресурсов и имеет большие времязатраты. Обычно в исследованиях используется гибридный подход в виде исследования сетевой информации и видеопотока, однако мы ориентируемся на информацию от видеокодека с видеопотоком.

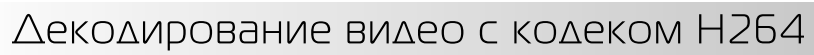 и артефакты потери пакетов}

Семейство сжатия видео MPEG, в частности MPEG 4 Part 10 (он же Н264) использует структуру гибридного кодирования. Межкадровое и внутрикадровое кодирование используются совместно для уменьшения размера видео. Помимо этого используются группы кадров (GOP)[2] для упрощения кодирования видео. Как при сжатии JPEG, блочное кодирование добавляет пространственные артефакты сжатия. При передаче по сети изображение может терять некоторые блоки, что приводит к артефактам потери пакетов, которые проявляются как квадратные области, заполненные цветным шумом.

\section{Типы ка $\Delta \mathrm{poB}$}

MPEG указывает [3], что кадры должны делиться на категории с названиями I, P и B (в MPEG 4 так же есть SI и SP, но они нужны для переключения между потоками и не представляют интереса в данной теме).

І-кадры - кадры, кодирующиеся независимо от других кадров. Процесс их кодирования похож на сжатие JPEG изображение, где сжимаются блоки размером 8 на 8 пикселей.

Р и В кадры представляют собой типы кадров, которые кодируются для удаления временной избыточности видео. Это достигается использованием предсказаний с оценкой и компенсаций движений. В результате в этих типах кадров анализируются и кодируются только изменения из ссылочных кадров. Причем макроблоки оценки и компенсации движения имеют размер 16 на 16 пикселей, в то время как макроблоки пространственных компенсаций имеют размер аналогичный I кадрам - 8 на 8 пикселей.

Р-кадры - кадры, использующие в качестве опорного предшествующие им I и Р кадры.

В-кадры - кадры, которые могут использовать в качестве опорных предыдущие и последующие кадры как I, так и Р типа. 


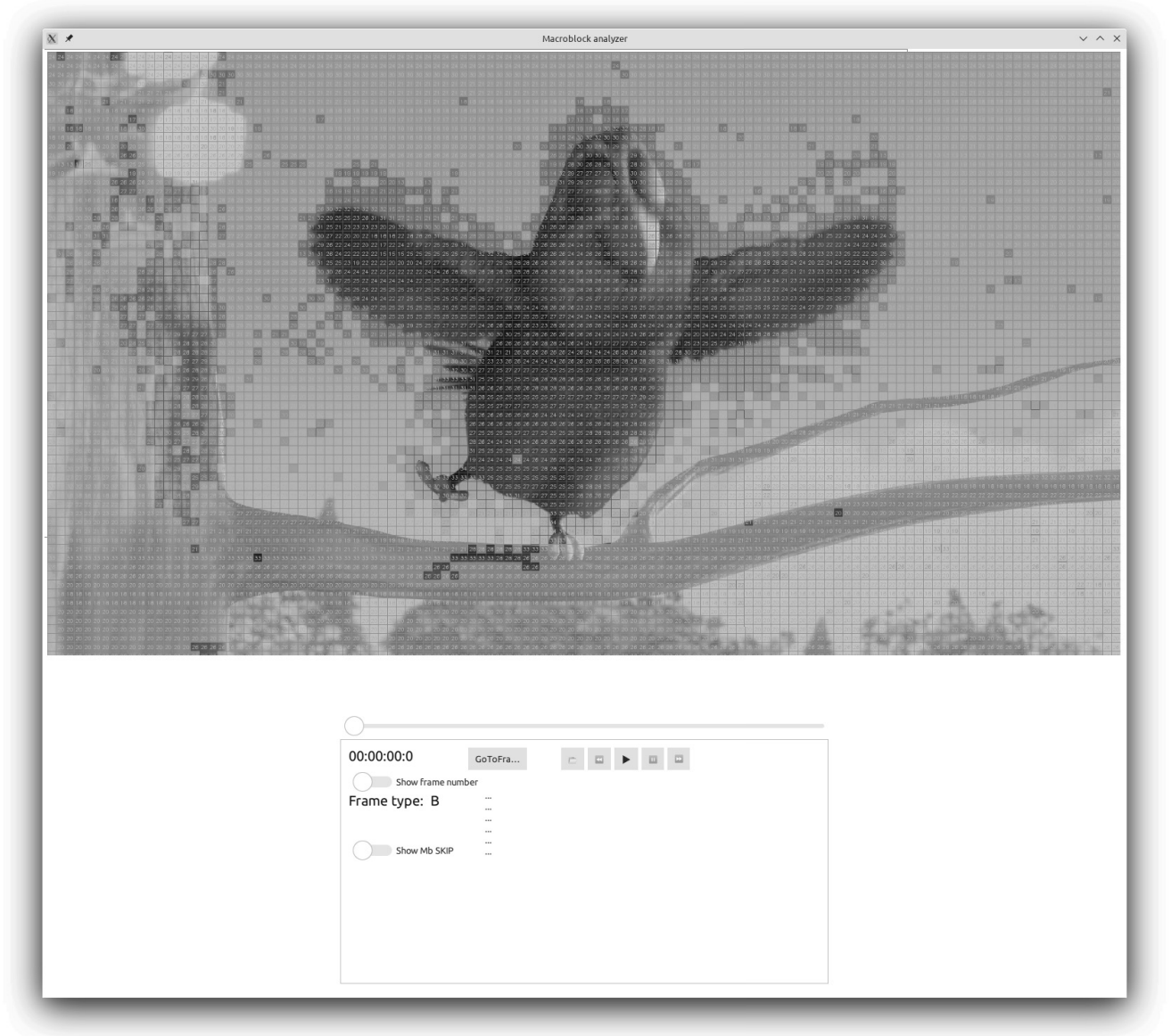

Рис. 2. Интерфейс разработанной программы для анализа макроблоков. Желтым выделены макроблоки типа «Skip»

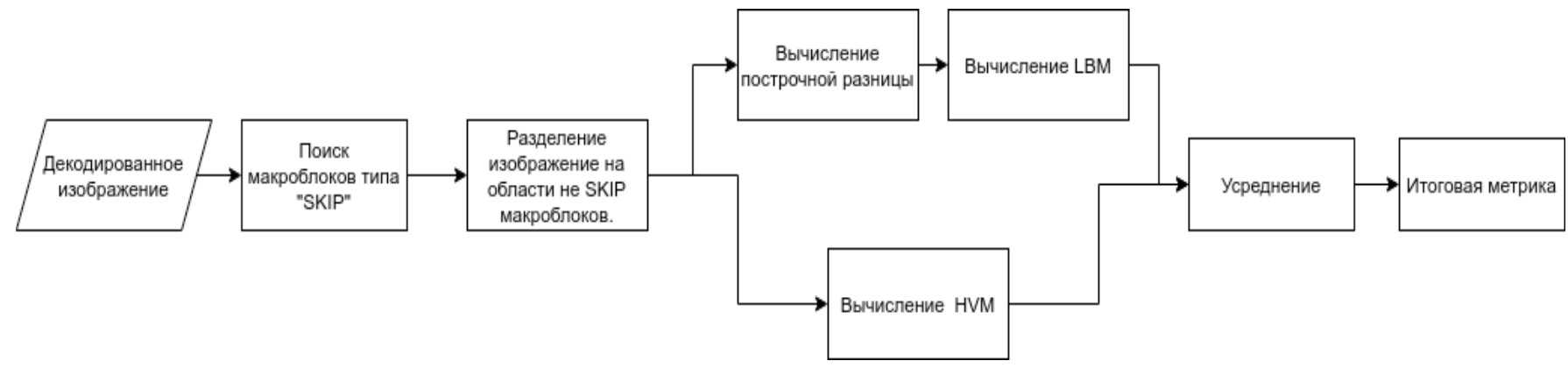

Рис. 3. Модифицированный алгоритм NPBM

\section{Таблича макроблоков}

Каждый кадр, вне зависимости от его типа, имеет таблицу макроблоков (Рисунок 1).

Каждый макроблок имеет базовый размер 8 на 8 пикселей и набор характеристик-типов. Среди типов макроблоков есть те, которые сообщают о реальном раз- мере блока, например увеличивающий его размер или наоборот, уменьшающей. Так же в каждом макроблоке содержится информация, откуда он взят. Сделано это по тому же принципу, по которому работают $\mathrm{P}$ и $\mathrm{B}$ кадры - макроблок может быть взят из следующего кадра или предыдущего, или вовсе иметь непосредственное представление. Так же он может не нести в себе информацию откуда он взят и как его обрабатывать, в данном 
Таблица 1. Времязатраты до и после учета Skip макроблоков

\begin{tabular}{|l|l|l|}
\hline С учетом SKIP Mb & NPBM & LeNet \\
\hline Оригинальный алгоритм & $00: 05: 27$ & $00: 12: 17$ \\
\hline
\end{tabular}

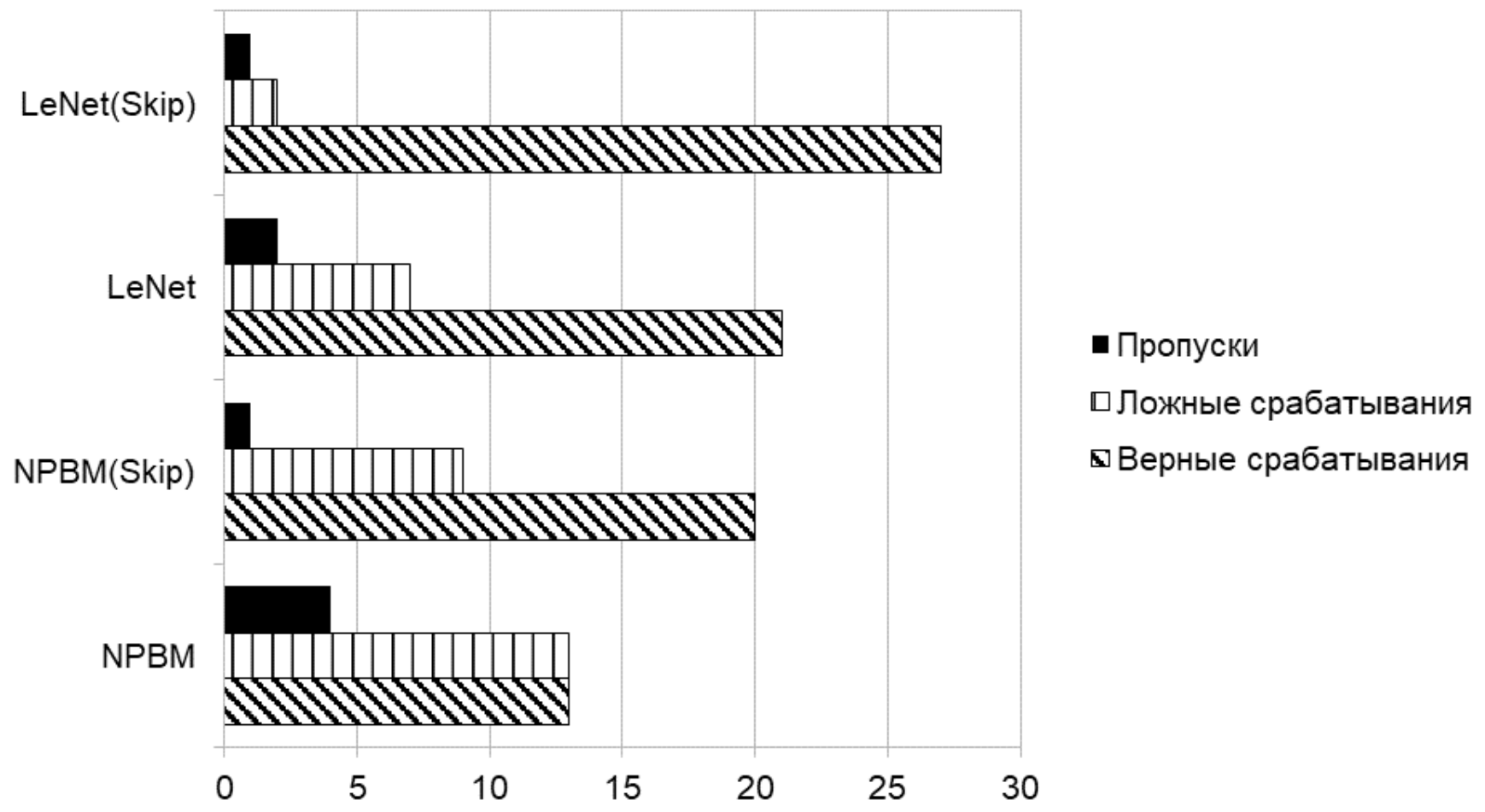

Рис. 4. Количество ложных срабатываний, верных и пропусков

случае макроблок имеет тип «Skip» [5], то есть «пропущено». Каждый макроблок обычно имеет сразу несколько таких типов, сообщающие, например о том, что он размером 16 на 16 пикселей, взят из предыдущего кадра и пропущен.

\section{Артефакты потери пакетов}

Для анализа поведения макроблоков в видео с артефактами потери пакетов было написано программное обеспечение [6], которое экспортирует таблицу макроблоков из кодека и выводит эту информацию на экран (Рисунок 2).

В ходе проведенного анализа видео, содержащего данные артефакты, было установлено, что они не попадают в макроблоки типа «Skip». В связи с этим было решено использовать это свойство в классических алгоритмах и в анализе нейронными сетями с целью уменьшить количество обрабатываемой информации и как следствие, снизить время обработки.
Для исследования было решено взять алгоритм NPBM[4] и модернизировать его для работы с учетом пропущенных макроблоков типа «Skip». Так же алгоритм учитывая кадры типа «|», которые не содержат в себе макроблоков данного типа. Итоговая схема данного алгоритма представлена на рисунке 3.

В качестве архитектуры для нейронной сети была выбрана LeNet[1], в силу её простоты, малого размера и достаточной сложности для определения типов артефактов на изображении.

Для тестирования алгоритмов было создано видео с размером кадра 1920 на 1080 пикселей. Результаты работы алгоритмов показаны на рисунках ниже.

Как видно из данных, приведенных выше, время работы алгоритмов уменьшилось, а количество верных срабатываний увеличилось. В связи с этим, данную особенность декодирования видеофайлов действительно можно применять на практике. 


\section{ЛИТЕРАТУРА}

1. LeCun, Y.; Boser, B.; Denker, J.S. “Backpropagation Applied to Handwritten Zip Code Recognition”. Neural Computation. 1 (4): [1998] c 541-551.

2. Keyframe, interframe \& Video compression | IBM Watson Media [Электронный ресурс].— Режим доступа: https://blog.video.ibm.com/streaming-video-tips/ keyframes-interframe-video-compression/\#keyframe, свободный. Дата обращения [04.11.2021]

3. ITU-T: Advanced video coding for generic audiovisual services [Tекст] 2016 г с. 101.

4. Hantao Liu, Ingrid Heynderickx. A No-Reference Perceptual Blockiness Metric [Tekcт] / ICASSP 2008, c. 865-868

5. SKIP macroblocks - Vcodex [Электронный ресурс].— Режим доступа: https://www.vcodex.com/news/skip-macroblocks/, свободный. Дата обращения [04.11.2021]

6. Устин A.M. Ratevandr/h264MacroblockAnalyzer [Электронный ресурс].— Режим доступа: https://github.com/Ratevandr/h264MacroblockAnalyzer, свободный. Дата обращения [05.11.2021]

(с) Устин Антон Михайлович ( ustinam95@уandex.ru),

Федосин Сергей Алексеевич ( fedosinsa@mrsu.ru ), Соколова Мария Сергеевна ( Manunia131313@mail.ru ).

Журнал «Современная наука: актуальные проблемы теории и практики»

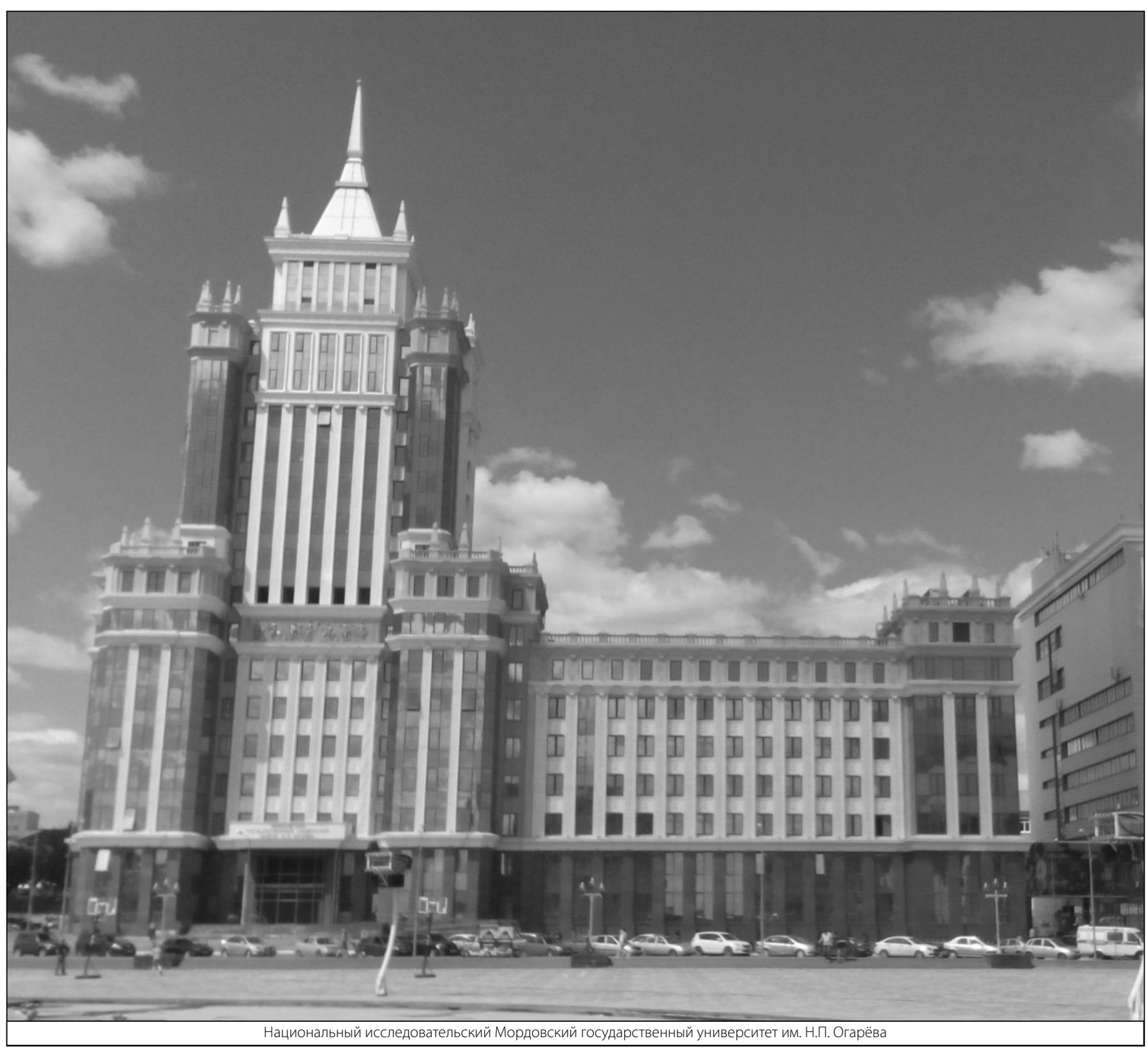

